> Ces dernières années, des études se sont focalisées sur l'existence d'une association entre la parodontite et la polyarthrite rhumatoïde (PR), suggérant l'implication du microbiote buccal dans le déclenchement de cette maladie autoimmune des articulations. D'un point de vue clinique, les deux pathologies reposent sur un processus inflammatoire qui conduit à une érosion osseuse. Elles font également intervenir une modification post-traductionnelle appelée citrullination. Dans le cas de la PR, la citrullination de certains sites protéiques par les peptidylarginine désiminases (PAD) aboutit à la production d'auto-anticorps. C'est la découverte d'une PAD exprimée par la bactérie Porphyromonas gingivalis qui a orienté de nombreuses études vers l'analyse d'une association entre ces deux pathologies. <

En France, $45 \%$ des adultes de plus de 40 ans sont atteints, au minimum, d'une parodontite localisée, une maladie inflammatoire chronique d'origine bactérienne touchant les tissus de soutien de la dent [1]. Depuis les années 1990, la médecine parodontale a émergé pour mettre notamment l'accent sur les conséquences liées à ces parodontopathies. Elle a en particulier identifié la parodontite comme étant un potentiel facteur de risque de maladies systémiques, telles que la polyarthrite rhumatoïde [2]. La polyarthrite rhumatoïde (PR) est une maladie auto-immune chronique touchant les articulations, qui se manifeste par une inflammation persistante au niveau de la membrane synoviale. D'un point de vue moléculaire, l'apparition des signes cliniques de la maladie est une conséquence de la citrullination de certains sites protéiques, qui peut déclencher une cascade d'événements aboutissant à la production d'anticorps anti-protéines citrullinées [3]. Un lien entre PR et parodontite a notamment été établi en raison de la

Vignette (Photo @ Canopé-CNDP-Universcience/MGEN/Inserm/EDUCAGRI).

\section{Peptidylarginine désiminases du microbiote buccal et polyarthrite rhumatoiide}

Marie Desclos-Theveniau, Martine Bonnaure-Mallet, Vincent Meuric

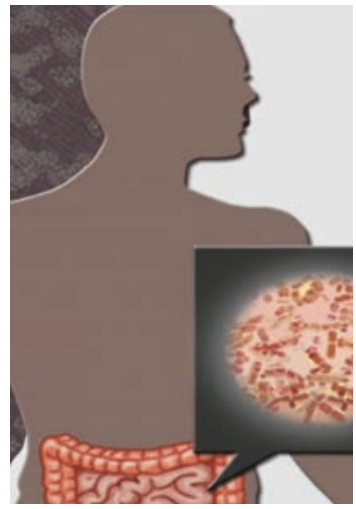

Inserm, INRAE, Univ Rennes 1, CHU de Rennes, Nutrition, Métabolismes et Cancer,

2 avenue du professeur Léon Bernard, 35043 Rennes, France. marie.theveniau@univ-rennesl.fr

découverte chez Porphyromonas gingivalis, une bactérie participant à l'établissement de la parondontite, d'une peptidylarginine désiminase, qui convertit l'arginine associée à un peptide (peptidylarginine) en peptidylcitrulline [4]. Dans cette revue, nous décrirons le rôle de cette citrullination des protéines dans les deux pathologies. Les différentes données scientifiques concernant le lien existant entre les deux maladies seront ensuite discutées.

\section{La citrullination des protéines}

La citrullination, ou désimination, est une modification post-traductionnelle des protéines qui consiste en la transformation de résidus peptidyl-arginyl en résidus peptidyl-citrullyl avec libération d'ammonium (Figure 1) [5]. Elle est réalisée par des enzymes appelées peptidylarginine désiminases ( $P A D$, pour protein-arginine deiminase, ou protein-L-arginine iminohydrolase, ou EC 3.5.3.15) (Figure 1) [5]. La citrullinisation joue un rôle important dans la kératinisation de la peau, la protection des neurones, la plasticité du système nerveux central, la régulation des gènes, mais aussi dans le fonctionnement du système immunitaire (Figure 2) [6]. En effet, c'est au cours de la réponse immunitaire innée, ou de la réponse inflammatoire, que l'hypercitrullination des histones permet la formation des NET (neutrophil extracellular trap) produits par les neutrophiles en réponse aux stimulus pathogènes, en particulier [7]. Ces NET (ou filets), constitués d'ADN et de protéines libérés par le neutrophile, sont capables de se lier aux bactéries pour les piéger et ainsi exercer une activité bactéricide extracellulaire à distance. Les histones liées à 


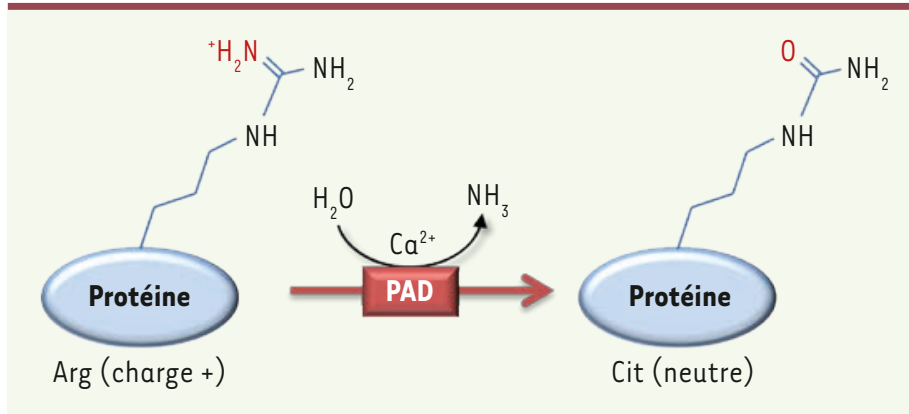

Figure 1. Processus de citrullination catalysé par les PAD humaines. PAD : peptidylarginine désiminase.

I'ADN seraient citrullinées, entraînant une modification de charge et une décondensation de I'ADN cellulaire [8]. Au cours des processus d'apoptose, de nécrose ou de NETose, un important flux d'ions calcium activerait les PAD qui citrullineraient de nombreuses protéines cytoplasmiques, nucléaires, membranaires et mitochondriales [6]. Dans le cas de la PR, ce sont des peptides présents au niveau des articulations, enrichis en citrulline, qui deviennent des auto-antigènes à l'origine de la réponse inflammatoire. La citrullination intervient également dans d'autres processus pathologiques, tels que la formation de tumeurs, le psoriasis, etc. (Figure 2) [6].

Chez l'homme, cinq enzymes PAD (PAD1, PAD2, PAD3, PAD4 et PAD6), sont codées par cinq gènes regroupés sur le chromosome 1 en un seul locus [5]. Ces isoformes sont assez bien conservées avec 44 à $57 \%$ d'identité en acides aminés (Tableau 1). Chez les procaryotes, ces enzymes n'ont été identifiées que chez Porphyromonas, plus particulièrement chez $P$. gingivalis, où l'homologue des PAD humaines a été nommé PPAD ( $P$. gingivalis peptidylarginine deiminase) [9]. La PPAD des Porphyromonas présente entre 30 et $40 \%$ d'identité avec les PAD humaines (Tableau I). Une comparaison entre PAD et PPAD est présentée dans le Tableau // $[6,10,11]$. Contrairement à ses homologues eucaryotes qui sont présentes dans le cytoplasme de la cellule, PPAD est sécrétée ou liée à la membrane externe de la paroi bactérienne. Son action ne dépend pas de la présence de calcium, et elle est active à un $\mathrm{pH}$ plus basique. Elle est capable de citrulliner des résidus arginines présents au sein des séquences protéiques ou au niveau de l'extrémité C-terminale de la protéine. Sa capacité d'autocitrullination reste à confirmer. Les substrats et la localisation de ces enzymes leur sont spécifiques (Tableau /I). PPAD peut en effet citrulliner des protéines bactériennes et des protéines de l'hôte, alors que les PAD n'agissent que sur des protéines humaines.

\section{Citrullination et parodontite chronique}

La parodontite chronique englobe un ensemble d'événements complexes, initiés par une colonisation bactérienne sous-gingivale, une inflammation chronique, une citrullination de protéines, et une dérégulation de la réponse immunitaire de l'hôte (Figure 3) [12]. La libération d'ammonium lors de la citrullination des protéines endogènes, protègerait $P$. gingivalis des attaques acides [13].
L'ammonium produit permettrait au milieu d'atteindre un $\mathrm{pH}$ optimal pour le fonctionnement de la PPAD, ce qui favoriserait la survie de $P$. gingivalis dans les poches parodontales [14]. PPAD n'aurait aucun impact sur la capacité de $P$. gingivalis à adhérer et à envahir les cellules épithéliales gingivales. Elle serait en revanche un modulateur puissant de la réponse immunitaire de l'hôte [15]. P. gingivalis dispose d'un large éventail de facteurs de virulence, parmi lesquels figurent les gingipaïnes Rgp (Arg-gingipain) et la PPAD [16]. Ces deux facteurs sont co-localisés sur la membrane externe de la paroi bactérienne ou dans des vésicules sécrétées. Pour traverser la paroi bactérienne, ils utilisent le système de sécrétion Por (PorSs), un mécanisme spécifique pour le transport des gingipaïnes [17]. Deux gingipaïnes spécifiques de l'arginine ont été identifiées chez $P$. gingivalis : RgpA, $\operatorname{RgpB}$. Ces deux enzymes clivent les protéines après les résidus arginines. Le fait que la PPAD citrulline préférentiellement les résidus arginines en position terminale des chaînes polypeptidiques, suggère l'existence d'un lien direct entre ces deux enzymes. Les produits issus de l'action des protéases Rgp sont ainsi des substrats pour la PPAD. Les actions successives des Rgp et de PPAD sur l'anaphylatoxine C5a, une glycoprotéine résultant de l'activation du complément, induit, par exemple, une perte de fonction de la protéine, ce qui empêche la vasodilatation des vaisseaux et le recrutement des neutrophiles [18]. L'inactivation des Rgp bactériennes a pour conséquence une diminution de la citrullination des protéines [19] et les niveaux d'expression et l'activité des Rgp sont augmentés en présence de PPAD [20, 21]. Les Rgp peuvent être, par ailleurs, citrullinées par la PPAD, ce qui suggère que la présence de résidus citrullines au sein de ces enzymes leur conférerait une protection contre une éventuelle auto-coupure aux niveaux des arginines qu'elles présentent [20].

La citrullination par PPAD du peptide LP9, ( ${ }_{107}$ RAWVAWRNR $_{115}$ ), un produit de la dégradation du lysozyme, connu pour agir comme peptide cationique antimicrobien, réduit son activité bactéricide [20]. PPAD empêcherait également la reconnaissance et la phagocytose de la bactérie par les neutrophiles, lui permettant de survivre dans le tissu gingival fortement enrichi en neutrophiles [20]. La citrullination par PPAD de l'histone $\mathrm{H} 3$, qui lui permet d'intégrer les NET produits par les neutrophiles, interroge sur les mécanismes exacts par lesquels PPAD participe à la formation de ces filets afin de capturer les bactéries [20]. P. gingivalis favoriserait donc la présence des cellules immunitaires tout en empêchant certaines de leurs activités, initiant 


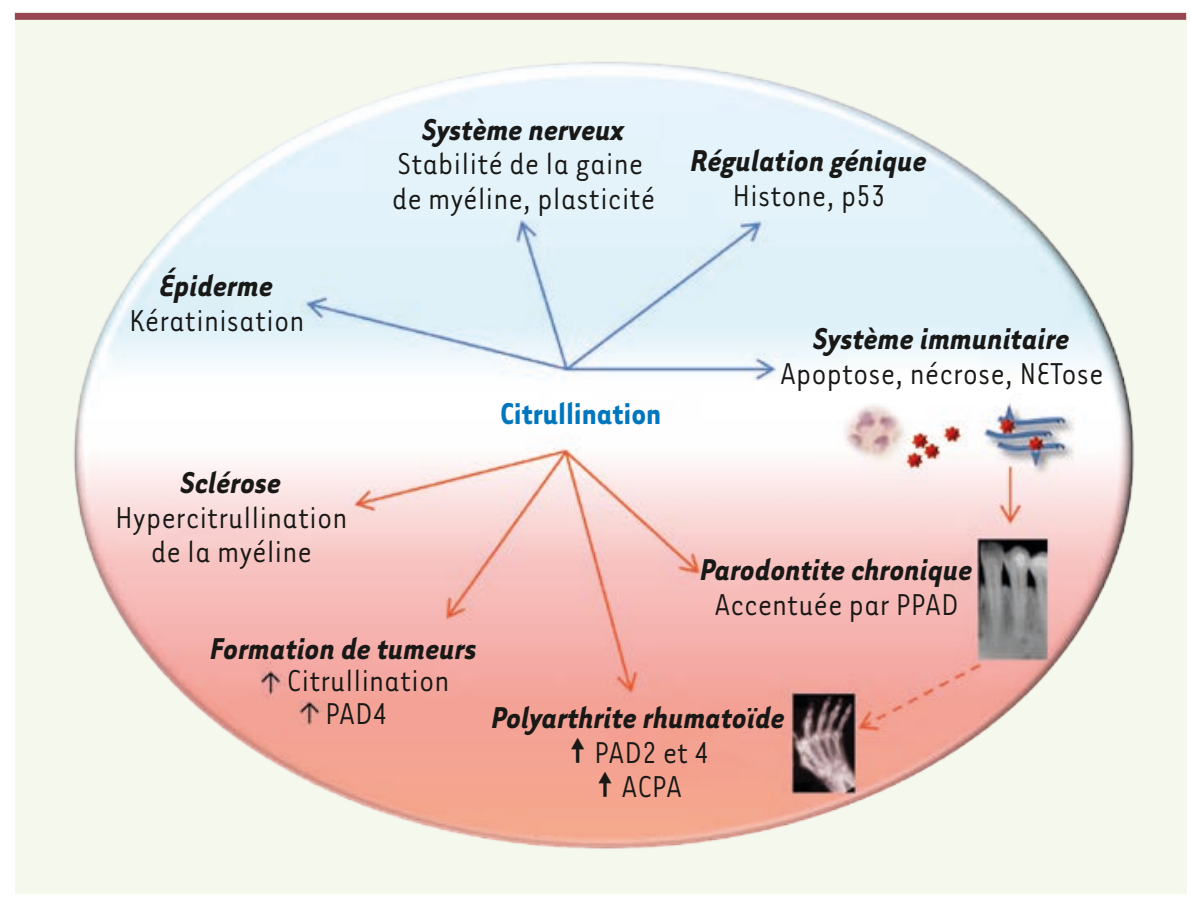

Figure 2. Processus de citrullination catalysé par les PAD. La citrullination intervient dans de nombreuses fonctions physiologiques (représentées par les flèches bleues). Un dysfonctionnement peut aboutir au développement de pathologies (représentés par les flèches rouges). NET : neutrophil extracellular trap, PAD : peptidylarginine désiminase, PPAD : peptidylarginine désiminase de Porphyromonas gingivalis.

sensibilité de l'enzyme au calcium [24]. Néanmoins, les mécanismes présidant à la production de ces auto-anticorps anti-PAD4 restent encore inconnus. Les ACPA sont des immunoglobulines d'isotype $G$ (IgG) qui sont produits sans besoin d'une éducation préalable des lymphocytes T (il s'agit donc d'une réponse

ainsi une réponse inflammatoire continue. L'ensemble de ces résultats posent ainsi de nombreuses questions quant à l'implication de PPAD dans les mécanismes de contournement des défenses mis en œuvre par $P$. gingivalis.

\section{Citrullination et polyarthrite rhumatoïde}

La physiopathologie de la polyarthrite rhumatoïde reste inconnue. II semble néanmoins que cette pathologie soit déclenchée par une combinaison de facteurs génétiques et environnementaux qui conduisent à une rupture de tolérance immunitaire. Cette maladie est caractérisée par une inflammation ayant pour origine une accumulation d'infiltrats leucocytaires dans la membrane synoviale des articulations, qui stimulent la production d'anticorps dirigés contre d'autres anticorps (facteurs rhumatoïdes) et d'anticorps plus spécifiques qui reconnaissent des peptides cycliques citrullinés (ACPA) comme des antigènes du non-soi [3]. Cette reconnaissance par les ACPA des épitopes citrullinés et la formation de complexes immuns intégrant des facteurs rhumatoïdes entretiennent un cercle vicieux à l'origine des lésions tissulaires aux niveaux des articulations. Les voies de signalisation impliquées dans la production des ACPA sont encore obscures. Cependant, il est établi que ces anticorps sont à l'origine de la persistance de l'inflammation synoviale en interagissant avec des protéines citrullinées localisées dans la membrane des cellules ou incorporées dans des NET [22]. L'analyse in vivo des véritables cibles des ACPA a permis d'identifier plusieurs candidats antigéniques : le fibrinogène, la vimentine, l' $\alpha$-énolase, le collagène de type II, mais aussi des histones contenues dans les NET [23]. L'existence d'auto-anticorps anti-PAD4, dont la présence est associée à la sévérité de la pathologie, a également été mise en évidence [24]. Ces anticorps seraient capables de se lier à PAD4 et de modifier sa conformation, augmentant ainsi la
T-indépendante). La production des auto-anticorps anti-PAD4 précède le développement d'une PR. L'association entre la protéine citrullinée et l'enzyme PAD4 (qui forme un complexe haptène-protéine porteuse) pourrait être reconnue par les lymphocytes $T$, et donc les activer [25]. Pourtant, les PAD sont des antigènes du soi. Elles devraient être tolérées par le système immunitaire. Les mécanismes par lesquels ces enzymes deviennent immunogènes et constituent des auto-antigènes dans cette maladie restent inconnus.

Depuis plusieurs années, le déclenchement de la PR par une infection bactérienne ou virale a été le sujet de plusieurs études. Les recherches se sont fondées sur le fait que l'inflammation provoquée par des bactéries ou des virus (virus d'Epstein-Barr, cytomégalovirus) pouvait entraîner une rupture de la tolérance immunitaire et ainsi provoquer l'apparition de maladies autoimmunes [2]. Les résultats obtenus dans ces études apparaissent cependant contradictoires. L'apparition précoce des auto-anticorps, bien avant la PR, a conduit à l'hypothèse qu'une inflammation, débutant dans les tissus muqueux, tels que les poumons, les intestins et le parodonte, pouvait, par voie systémique, atteindre les articulations [26].

\section{La citrullination : lien entre polyarthrite rhumatoïde et parodontite chronique}

Malgré des étiologies différentes, la parodontite et la PR sont des maladies multifactorielles qui partagent de nombreuses caractéristiques. Elles sont toutes deux le 


\begin{tabular}{|ccccccc}
\hline Identité (\%) & PAD1 & PAD2 & PAD3 & PAD4 & PAD6 & PPAD \\
\hline PAD1 & 100 & 51 & 57 & 56 & 44 & 45 \\
\hline PAD2 & 100 & 51 & 50 & 44 & 35 \\
\hline PAD3 & & 100 & 55 & 43 & 44 \\
\hline PAD4 & & 100 & 100 & 29 \\
\hline PAD6 & & & & 34 \\
\hline PPAD & & & & 100 \\
\hline
\end{tabular}

Tableau I. Conservation entre les différentes PAD humaines et PPAD. Les pourcentages d'identité en acides aminés ont été calculés après alignement des séquences protéiques sur Blastp (https://blast.ncbi.nlm.nih.gov/Blast.cgi?PAGE=Proteins). PAD : peptidylarginine désiminase; PPAD : peptidylarginine désiminase de Porphyromonas gingivalis.

\begin{tabular}{|c|c|}
\hline PPAD & PAD humaines \\
\hline Un seul isotype & PAD 1-4 et PAD6 \\
\hline Liée à la membrane externe de la paroi bactérienne & PAD 1 -3 et PAD6 dans le cytoplasme \\
\hline ou présente dans des vésicules sécrétées & PAD 4 et 2 dans le cytoplasme et le noyau \\
\hline Citrulline les résidus Arg internes ou en C-terminal & Citrullinent les résidus Arg internes \\
\hline Calcium indépendante & Calcium dépendantes \\
\hline Autocitrullination??? & Autocitrullination \\
\hline Substrats : & Substrats : \\
\hline $\begin{array}{l}\text { Protéines bactériennes : gingipaïnes, protéine de fimbriline Mfal } \\
\text { Protéines humaines : fibrinogène, énolase, histone, vimentine, fac- } \\
\text { teur de croissance épidermique (EGF), C5a, produit de dégradation } \\
\text { du lysosome LP9 }\end{array}$ & $\begin{array}{l}\text { Protéines humaines : vimentine, fibrinogène, myéline, actines, } \\
\text { histones, fibrine, filaggrine, trichohyaline, facteur inducteur } \\
\text { d'apoptose, énolase }\end{array}$ \\
\hline
\end{tabular}

Tableau II. Comparaison entre les différentes PAD humaines et PPAD. Arg : arginine; PAD : peptidylarginine désiminase ; PPAD : peptidylarginine désiminase de Porphyromonas gingivalis (d'après $[10,11]$ ).

résultat de réactions inflammatoires chroniques localisées, auxquelles participent un ensemble de cytokines (TNF- $\alpha$ [tumor necrosis factor alpha], IL[interleukine]-6 et IL-17) dont la circulation dans le sang est bidirectionnelle, du parodonte aux articulations et vice versa [2]. Elles induisent également une augmentation de l'activité des métalloprotéinases de la matrice collagénique ainsi que celle d'autres enzymes (élastase, protéases à cystéines, des enzymes produites par les neutrophiles). Elles sont aussi à l'origine de la surexpression du système RANK/RANKL/OPG ${ }^{1}$, responsable de lésions irréversibles sur les tissus riches en collagène (gencive, ligament parodontal et os alvéolaire pour la parodontite, os sous-chondral et cartilage pour la PR) [27]. La parodontite et la PR partagent également de nombreux facteurs de risque environnementaux (tabac, etc.) et génétiques (dont le complexe majeur d'histocompatibilité de classe I, HLA-DR, etc.) [11].

${ }^{1}$ Le récepteur membranaire receptor activator of NF- $k B$ (RANK) et son ligand (RANKL) jouent un rôle fondamental dans la régulation du remodelage osseux. L'ostéoprotégérine (OPG) bloque ce processus en interagissant avec RANKL.
Ces dernières années, de nombreuses analyses ont tenté de montrer que la parodontite pouvait précéder le développement de la PR [11, 27]. La théorie qui a émergé de ces études suppose que le parodonte enflammé serait le lieu de production des ACPA, en réponse à des processus d'hypercitrullination de protéines réalisés par les PAD endogènes et la PPAD bactérienne.

II reste cependant difficile d'établir une relation entre les deux pathologies, malgré des caractéristiques communes et des liens suggérés par les données actuelles: 1) la prévalence élevée de la maladie parodontale chez les patients atteints de PR et vice-versa. De nombreuses études cas-témoins montrent en effet que la maladie parodontale est plus fréquente chez les patients atteints de PR, comparativement aux individus en bonne santé [11]. Et réciproquement, la prévalence de la PR est plus élevée chez les patients ayant une parodontite. Dans l'étude la plus importante menée 

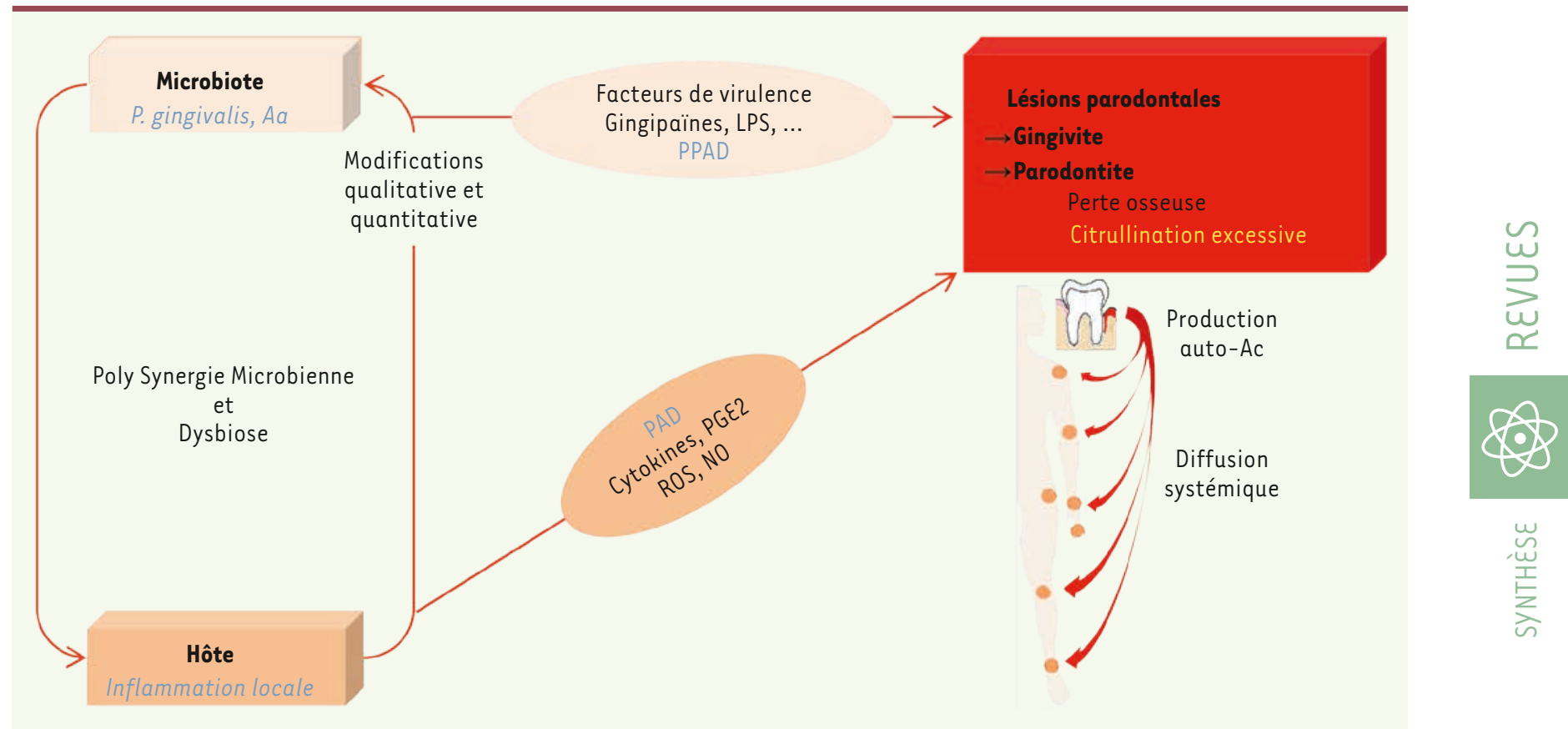

Figure 3. Séquence d'évènements lors de la mise en place de la parodontite. La formation de lésions parodontales est provoquée par une dysbiose sous-gingivale (altération qualitative et quantitative de la flore bactérienne) ayant provoqué et amplifié une réponse immunitaire inflammatoire locale. Ces atteintes parodontales sont le résultat d'une action concommitante des facteurs de virulence et des médiateurs de l'inflammation. La citrullination excessive des protéines par les PAD humaines et bactérienne (PPAD) fait partie des processus mis en jeu à l'origine de la production d'auto-anticorps pouvant diffuser de manière systémique. Aa : Aggregatibacter actinomycetemcomitans ; Ac : anticorps ; LPS : lipopolysaccharides; NO: monoxyde d'azote; PAD : peptidylarginine désiminase; P. gingivalis : Porphyromonas gingivalis ; PGE2 : prostaglandine $\varepsilon 2$; PPAD : peptidylarginine désiminase de Porphyromonas gingivalis; ROS : espèces réactives de l'oxygène.

jusqu'à ce jour (plus de 13000 cas de PR et 130000 témoins), une association significative a été mise en évidence entre les deux pathologies [28]. Toutefois, d'autres études ne confirment pas ces conclusions $[10,11]$. Les raisons expliquant de tels résultats contradictoires peuvent être le manque de critères de classification spécifiques associés à la parodontite, la taille des cohortes de patients, l'absence de données concernant les facteurs de confusion et les différences de design entre études. À noter que la prévalence de la PR est trois fois plus importante chez les femmes [11]. Il existe d'autres facteurs (hormones stéroïdiennes, marqueurs génétiques, etc.) et mécanismes spécifiques à chacune des pathologies, autres que la citrullination, qui sont à prendre en compte ;

2) la présence de taux élevés d'anticorps anti-P. gingivalis dans la PR [10]. II est difficile d'évaluer la pertinence des différentes études en raison de l'absence d'analyses standardisées pour mesurer les niveaux d'anticorps. Cependant, deux méta-analyses regroupant un nombre conséquent de publications ont établi une corrélation entre les niveaux d'anticorps anti-P. gingivalis et d'ACPA [29]. Contrairement aux anticorps anti-P. gingivalis, les anticorps dirigés contre $\operatorname{RgpB}$, ne sont pas corrélés à la PR [29]. Toutefois, des concentrations élevées d'anticorps anti-RgpB ont été détectées avant l'apparition clinique de la PR [31] ; 3) des symptômes cliniques de PR aggravés chez les souris et les rats infectés par P. gingivalis. Un certain nombre d'études ont montré un afflux massif de leucocytes, une accumulation d'ostéoclastes et des lésions articulaires plus sévères après une infection à $P$. gingivalis $[11,32]$. Cependant, la validité de tels modèles est contestée. Les animaux produisent effectivement des protéines citrullinées au niveau de la synoviale, mais celles-ci ne présentent cependant pas les épitopes spécifiques observés au cours de la PR chez l'homme [10] ;

4) les effets bénéfiques des thérapeutiques sur l'évolution de ces pathologies. Un traitement parodontal non chirurgical permettrait d'améliorer les signes et les symptômes de la PR [11,33]. L'impact de cette thérapeutique sur la PR semble être plus important chez les patients présentant une inflammation systémique sévère [34]. Le traitement diminue l'activité de la $P R$, avec de faibles taux de facteur rhumatoïde et d'ACPA, mais aussi d'anticorps anti-P. gingivalis, chez les patients simultanément atteints de $P R$ et de parodontite chronique $[35,36]$. Inversement, le ralentissement précoce de la PR par des médicaments antirhumatismaux peut également limiter les lésions parodontales [11];

5) de l'ADN de $P$. gingivalis a été détecté dans le liquide synovial de patients atteints de PR, ce qui n'est pas le cas chez des patients en bonne santé [37]. La présence d'une PPAD unique chez Porphyromonas est l'élément 
clé qui a déclenché de nombreuses études. Cette enzyme peut citrulliner des protéines de l'hôte, dont l'énolase et le fibrinogène, deux des principaux auto-antigènes candidats dans la PR [19]. Elle est à l'origine de nouveaux peptides constituant ainsi un pool d'épitopes induisant la production des ACPA au niveau gingival. Une analyse du «citrullome» par spectrométrie de masse révèle que 6 protéines citrullinées qui sont détectées dans le tissu parodontal le sont également dans le fluide synovial de patients atteints de PR [38]. Les peptides citrullinés retrouvés au niveau du tissu parodontal des patients atteints de parodontite pourraient ainsi représenter des auto-antigènes dans la PR. Toutefois, le peu de peptides identifiés, le faible nombre d'échantillons examinés, ainsi que les limites des techniques employées, ne permettent pas de conclure quant au lien entre les deux pathologies;

6) I'implication d'un autre agent parodontopathogène. P. gingivalis n'était pas la seule espèce bactérienne expliquant le lien entre les deux pathologies. Aggregatibacter actinomycetemcomitans $(A a)$ serait également à l'origine d'une hypercitrullination dans les neutrophiles via la sécrétion de la leukotoxine A [39]. Cette toxine induirait la formation de pores dans la membrane des cellules, permettant un influx de calcium intracellulaire qui activerait les PAD. Une telle activité de PAD a été identifiée dans le fluide gingival [40]. Des recherches complémentaires sont néanmoins nécessaires pour comprendre le potentiel rôle des bactéries dans l'initiation et l'évolution de la maladie.

\section{Conclusion}

La citrullination fait partie des modifications post-traductionnelles les moins étudiées à ce jour. La mise en évidence de son implication dans la séquence d'évènements participant à la mise en place de la maladie parodontale est très récente. De nombreuses interrogations restent sans réponse. Bien que certaines études montrent le rôle de PPAD comme facteur de virulence bactérien, sa participation dans la maladie parodontale reste encore à préciser. Ces dernières années, de nombreuses analyses ont tenté de montrer que le parodonte enflammé pouvait être le lieu de production d'ACPA issus du processus d'hypercitrullination via les PAD et la PPAD. La validation d'un tel modèle nécessitera d'autres études afin d'identifier les mécanismes mis en jeu et évaluer avec exactitude l'implication du microbiote buccal dans le déclenchement de la PR. $\diamond$

\section{SUMMARY}

Protein arginine deiminase of oral microbiome plays a causal role in the polyarthritis rheumatoid initiating

In the last decade, the association between the periodontitis and rheumatoid arthritis (RA) has been established, suggesting that oral microbiome plays a causal role by initiating this chronic autoimmune inflammatory disease of articulation. Both pathogenesis are similar in term of chronic inflammation, tissue breakdown and bone resorption. Molecular aspects have also revealed that citrullination, a posttranslational modification catalyzed by peptidyl-arginine deiminases (PADs), is involved in both diseases. For RA, citrullinated proteins production leads to the synthesis the of anti-citrullinated protein antibodies triggering the loss of immune tolerance. In humans, five PADs have been identified. Recently, studies have found that only Porphyromonas species possess PAD. Thus, a major periodontal pathogen, Porphyromonas gingivalis, is able to generate citrullinated epitopes, and could consequently induce anti-citrullinated protein antibodies. In this review, citrullination process, periodontitis and RA are described to put them in relation with molecular, clinical and epidemiological studies establishing the association between periodontitis and RA. $\diamond$

\section{LIENS D'INTÉRÊT}

Les auteurs déclarent n'avoir aucun lien d'intérêt concernant les données publiées dans cet article.

\section{RÉFÉRENCES}

1. Bourgeois D, Bouchard P, Mattout C. Epidemiology of periodontal status in dentate adults in France, 2002-2003. J Periodont Res 2007 ; $42: 21-7$.

2. Kumar PS. From focal sepsis to periodontal medicine: a century of exploring the role of the oral microbiome in systemic disease. J Physiol (Lond) 2017 ; $595: 465-76$.

3. Sebbag M, Chapuy-Regaud S, Auger I, et al. Clinical and pathophysiological significance of the autoimmune response to citrullinated proteins in rheumatoid arthritis. Joint Bone Spine 2004 ; 71 : 493-502.

4. McGraw WT, Potempa J, Farley D, et al. Purification, characterization, and sequence analysis of a potential virulence factor from Porphyromonas gingivalis, peptidylarginine deiminase. Infect Immun 1999; 67 : 3248-56.

5. Vossenaar ER, Zendman AJW, Venrooij WJ van, et al. PAD, a growing family of citrullinating enzymes: genes, features and involvement in disease. Bioessays 2003; 25 : 1106-18.

6. Baka Z, György B, Géher P, et al. Citrullination under physiological and pathological conditions. Joint Bone Spine 2012 ; 79 : 431-6.

7. Branzk N, Lubojemska A, Hardison $S E$, et al. Neutrophils sense microbe size and selectively release neutrophil extracellular traps in response to large pathogens. Nat Immunol $2014 ; 15$ : 1017-25.

8. Li P, Li M, Lindberg MR, et al. PAD4 is essential for antibacterial innate immunity mediated by neutrophil extracellular traps. J Exp Med 2010 ; 207 : 1853-62.

9. Gabarrini G, Chlebowicz MA, Vega Quiroz ME, et al. Conserved citrullinating exoenzymes in porphyromonas species. J Dent Res 2018 ; 97 : 556-62.

10. Gómez-Bañuelos $\varepsilon$, Mukherjee A, Darrah $\varepsilon$, et al. Rheumatoid arthritisassociated mechanisms of Porphyromonas gingivalis and Aggregatibacter actinomycetemcomitans. J Clin Med $2019 ; 8$ : 1309.

11. Potempa J, Mydel P, Koziel J. The case for periodontitis in the pathogenesis of rheumatoid arthritis. Nat Rev Rheumatol 2017 ; $13: 606-20$.

12. Ancuta $C$, lordache $C$, Ancuta $\varepsilon$, et al. Rheumatoid arthritis and periodontal disease: a complex interplay, new developments in the pathogenesis of rheumatoid arthritis. Lazaros I. Sakkas, Intech0pen. doi: 10.5772/65863. https://www.intechopen.com/books/new-developments-in-thepathogenesis-of-rheumatoid-arthritis/rheumatoid-arthritis-andperiodontal-disease-a-complex-interplay

13. Casiano-Colón A, Marquis RE. Role of the arginine deiminase system in protecting oral bacteria and an enzymatic basis for acid tolerance. Appl Environ Microbiol 1988 ; 54 : 1318-24.

14. Rodríguez SB, Stitt BL, Ash DE. Expression of peptidylarginine deiminase from Porphyromonas gingivalis in Escherichia coli: enzyme purification and characterization. Arch Biochem Biophys 2009 ; 488 : 14-22.

15. Aliko A, Kamińska M, Bergum B, et al. Impact of Porphyromonas gingivalis peptidylarginine deiminase on bacterial biofilm formation, epithelial cell invasion, and epithelial cell transcriptional landscape. Sci Rep 2018 ; 8 : 14144.

16. How KY, Song KP, Chan KG. Porphyromonas gingivalis: an overview of periodontopathic pathogen below the gum line. Front Microbiol $2016 ; 7$. 


\section{RÉFÉRENCES}

17. Sato K, Yukitake H, Narita Y, et al. Identification of Porphyromonas gingivalis proteins secreted by the Por secretion system. FEMS Microbiol Lett 2013 ; 338 : 68-76.

18. Bielecka $\varepsilon$, Scavenius $C$, Kantyka T, et al. Peptidyl arginine deiminase from Porphyromonas gingivalis abolishes anaphylatoxin C5a activity. J Biol Chem $2014 ; 289: 32481-7$.

19. Wegner N, Wait R, Sroka A, et al. Peptidylarginine deiminase from Porphyromonas gingivalis citrullinates human fibrinogen and $\alpha$-enolase: Implications for autoimmunity in rheumatoid arthritis. Arthritis Rheum $2010 ; 62$ : 2662-72

20. Stobernack T, Espina M du T, Mulder LM, et al. A Secreted bacterial peptidylarginine deiminase can neutralize human innate immune defenses. mBio 2018 ; 9 : e01704-18.

21. Stobernack T, Glasner $C$, Junker $S$, et al. Extracellular proteome and citrullinome of the oral pathogen Porphyromonas gingivalis. J Proteome Res 2016 ; 15 : 4532-43.

22. Khandpur R, Carmona-Rivera C, Vivekanandan-Giri A, et al. NETs are a source of citrullinated autoantigens and stimulate inflammatory responses in rheumatoid arthritis. Sci Transl Med 2013 ; $5: 178 \mathrm{ra} 40-178 \mathrm{ra} 40$

23. Wang $F$, Chen FF, Gao WB, et al. Identification of citrullinated peptides in the synovial fluid of patients with rheumatoid arthritis using LC-MALDI-TOF/TOF. Clin Rheumatol 2016 ; 35 : 2185-94.

24. Darrah $\varepsilon$, Giles JT, Ols ML, et al. Erosive rheumatoid arthritis is associated with antibodies that activate PAD4 by increasing calcium sensitivity. Sci Transl Med $2013 ; 5: 186 \mathrm{ra} 65$.

25. Roudier J, Balandraud N, Auger I. Anti PAD autoimmunity and rheumatoid arthritis. Joint Bone Spine $2018 ; 85: 659-61$.

26. Chen J, Wright K, Davis JM, et al. An expansion of rare lineage intestinal microbes characterizes rheumatoid arthritis. Genome Med 2016; $8: 43$

27. Oliveira Ferreira R de, Brito Silva R de, Magno MB, et al. Does periodontitis represent a risk factor for rheumatoid arthritis? A systematic review and meta-analysis. Ther Adv Musculoskelet Dis $2019 ; 11: 1759720 \times 19858514$

28. Chen $\mathrm{HH}$, Huang $\mathrm{N}$, Chen $\mathrm{YM}$, et al. Association between a history of periodontitis and the risk of rheumatoid arthritis: a nationwide, population-based, case-control study. Ann Rheum Dis 2013 ; 72 : 1206-11.

29. Bae SC, Lee YH. Association between anti-Porphyromonas gingivalis antibody, anti-citrullinated protein antibodies, and rheumatoid arthritis. Z Rheumatol $2018 ; 77: 522-32$

30. Bender P, Bürgin WB, Sculean A, et al. Serum antibody levels against Porphyromonas gingivalis in patients with and without rheumatoid arthritis: a systematic review and meta-analysis. Clin Oral Investig $2017 ; 21: 33-42$.
31. Johansson L, Sherina $\mathrm{N}$, Kharlamova $\mathrm{N}$, et al. Concentration of antibodies against Porphyromonas gingivalis is increased before the onset of symptoms of rheumatoid arthritis. Arthritis Res Ther 2016; $18: 201$.

32. Marchesan JT, Gerow EA, Schaff R, et al. Porphyromonas gingivalis ora infection exacerbates the development and severity of collagen-induced arthritis. Arthritis Res Ther $2013 ; 15$ : R186.

33. Kaur $S$, Bright $R$, Proudman $S M$, et al. Does periodontal treatment influence clinical and biochemical measures for rheumatoid arthritis? A systematic review and meta-analysis. Semin Arthritis Rheum $2014 ; 44$ : 113-22.

34. Monsarrat $P$, Vergnes JN, Cantagrel A, et al. Effect of periodontal treatment on the clinical parameters of patients with rheumatoid arthritis: study protocol of the randomized, controlled ESPERA trial. Trials $2013 ; 14: 253$.

35. Ortiz P, Bissada NF, Palomo L, et al. Periodontal therapy reduces the severity of active rheumatoid arthritis in patients treated with or without tumor necrosis factor inhibitors. J Periodontol $2009 ; 80: 535-40$

36. Okada M, Kobayashi T, Ito $S$, et al. Periodontal treatment decreases levels of antibodies to Porphyromonas gingivalis and citrulline in patients with rheumatoid arthritis and periodontitis. J Periodontol $2013 ; 84:$ e 74-84.

37. Moen K, Brun JG, Valen M, et al. Synovial inflammation in active rheumatoid arthritis and psoriatic arthritis facilitates trapping of a variety of oral bacterial DNAs. Clin Exp Rheumatol 2006 ; 24 : 656-63.

38. Schwenzer A, Quirke AM, Marzeda AM, et al. Association of distinct fine specificities of anti-citrullinated peptide antibodies with elevated immune responses to Prevotella intermedia in a subgroup of patients with rheumatoid arthritis and periodontitis. Arthritis Rheum (Hoboken NJ) 2017 ; 69 : 2303-13.

39. Konig MF, Abusleme L, Reinholdt J, et al. Aggregatibacter actinomycetemcomitans-induced hypercitrullination links periodontal infection to autoimmunity in rheumatoid arthritis. Sci Transl Med $2016 ; 8$ : 369ral76.

40. Harvey GP, Fitzsimmons TR, Dhamarpatni A, et al. SSK, et al. Expression of peptidylarginine deiminase -2 and -4 , citrullinated proteins and anticitrullinated protein antibodies in human gingiva. J Periodontal Res 2013 $48: 252-61$

TIRÉS À PART

M. Desclos-Theveniau

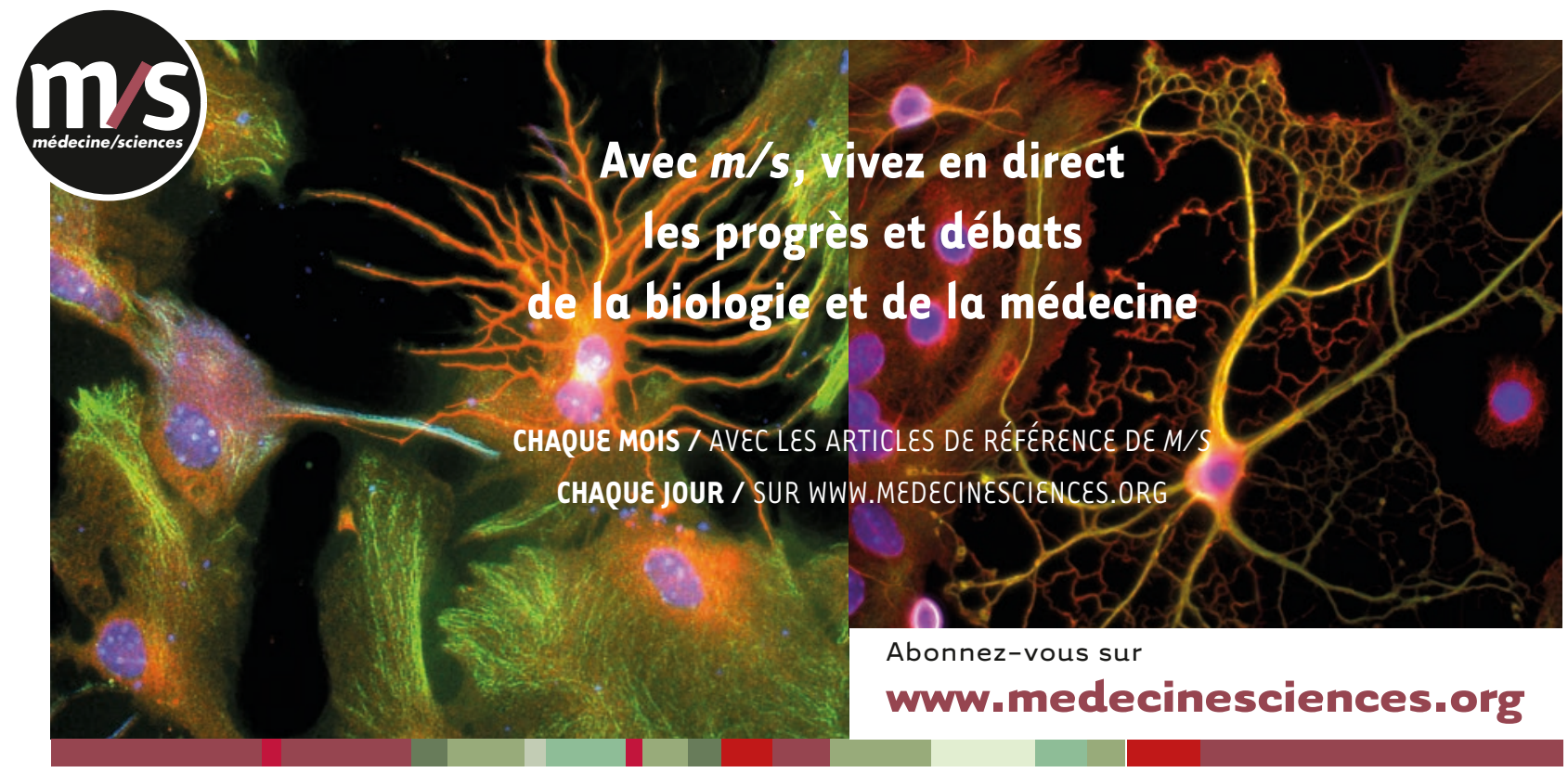

Retrouvez toutes les Actualités de la Myologie sur les sites de :

la Société Française de Myologie

www.sfmyologie.org

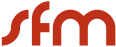

la filière de santé neuromusculaire FILNEMUS

www.filnemus.fr 